\title{
REPTILIA VS. SAUROPSIDA REPTILIA VS. SAUROPSIDA
}

\author{
Oscar A. Flores Villela ${ }^{1 *}$ \\ ${ }^{1}$ Museo de Zoología, Facultad de Ciencias, Universidad Nacional Autónoma de México. CDMX, México. \\ "correspondence: ofvq@unam.mx \\ Received: 2021-03-07. Accepted: 2021-04-26.
}

Este pequeño ensayo obedece a una controversia que he tenido por algunos años con algunos colegas herpetólogos acerca del uso de la palabra Sauropsida como sustituto de Reptilia. Desde mi punto de vista, esto ha conducido a algunos grupos de trabajo a sustituir el ampliamente usado y conocido término Reptilia por el de Sauropsida. A mi parecer, es un error que no debe perpetuarse. He revisado la literatura hasta donde me ha sido posible, considerando que las bibliotecas están cerradas y no he tenido acceso a ciertas obras que no están disponibles en PDF o que no poseo en mi biblioteca personal. Creo que este ensayo resume los usos que se le ha dado a la categoría de Sauropsida, y aclara si se la ha propuesto para remplazar a la palabra Reptilia. Mi interpretación de esta controversia se resume aquí. También sugiero cuál de estos términos se debe usar y bajo qué condiciones del conocimiento de los organismos familiares para todos conocidos como reptiles. A lo largo del escrito utilizo término y categoría para referirme a Reptilia y Sauropsida en diferentes contextos; a propósito no utilizo categorías linneanas, sino grupos monofiléticos o parafiléticos aclarándolo en cada caso. Sirva esta explicación a manera de introducción de este ensayo.

El término Sauropsida fue propuesto originalmente Huxley (1864), en una serie de lecciones de anatomía comparada que el mismo ofreció en 1863 (Huxley, 1864, v.). En esta publicación introdujo el término "Sauroid" para referirse a las similitudes anatómicas entre reptiles y aves en su clasificación de los vertebrados (ver también Switek, 2010), aunque en el texto también menciona a los Sauropsida (p.220), al referirse a la estructura del cráneo de los reptiles y aves. En resumen, Huxley $(1864,1869,1872)$ utilizó el término "saurópsidos" para referirse a dos grupos de vertebrados: reptiles y aves, y no como substituto de Reptilia. En todo caso, como concluye Switek (2010, p. 255), Huxley propuso el término Sauropsida como un sistema anatómico y no evolutivo, ya que se habían derivado de una "superestructura". Por ejemplo, Huxley (1870), en su clasificación de Dinosauria, no utilizó Sauropsida, refiriéndose a Reptilia en su disertación.
Baur (1887) utilizó la categoría Sauropsida, para incluir a los Crocodylia, Dinosauria, Aves y Ornithosauria, como un subgrupo de reptiles. Goodrich (1916) propuso que los Reptilia no son un grupo monofilético, conteniendo dos subgrupos: Theropsida (incluyendo mamíferos) y Sauropsida (incluyendo aves). Este esquema fue seguido por Watson (1957). Pearse (1947) en su lista de nombres de categorías zoológicas mayores (phyla, clases y órdenes) consideró a Sauropsida, dentro del cual incluyó a Reptilia, Aves y Mammalia. Sin embargo, Pearse (1947) acreditó el término de Sauropsida a Conybeare, 1823 (William Daniel Conybeare, geólogo británico, no encontré dicha publicación). De la lista de taxones publicada por Pearse, aparentemente se publicaron cuatro ediciones. Yo solo tuve acceso a tres, de las cuales únicamente la segunda (1947) menciona el término Sauropsida y su contenido de grupos.

Posteriormente, Gauthier et al. (1988), con un enfoque cladístico propusieron una filogenia de los Amniota, incluyendo a dos clados Mammalia y Reptilia; el clado Reptilia incluye a Chelonia + Sauria [Lepidosauria + Archosauria]). Esta filogenia (Fig. 1) fue la referencia por varios años (por ejemplo ver revisión en Flores Villela y Navarro 1993). Loconte (1990) cuestionó el trabajo de Gauthier et al (1988), bajo los argumentos que el uso de fósiles es un problema en la nomenclatura de taxones recientes y en la reconstrucción filogenética; que la definición original de Reptilia no incluye Aves; que Reptilia es parafilético (siguiendo a Hennig, 1968, 1975) y que el término adecuado para el grupo monofilético que incluya Reptilia y Aves es Sauropsida en el sentido de Goodrich (1916). Kluge (1990) rebatió las objeciones de Loconte, con los siguientes argumentos: los taxones nombrados en el trabajo de Gauthier et al. (1988) representan grupos monofiléticos y como tales están definidos en esos términos; el contenido del taxón Reptilia ha variado con el tiempo; y Sauropsida también ha tenido varios significados. No obstante, se puede mantener la monofilia de Reptilia independientemente de su contenido de taxones (Gauthier et al., 1988; Laurin \& Reisz, 1995; Dominguez \& Wheeler, 1997; Modesto \& Anderson, 2004). 


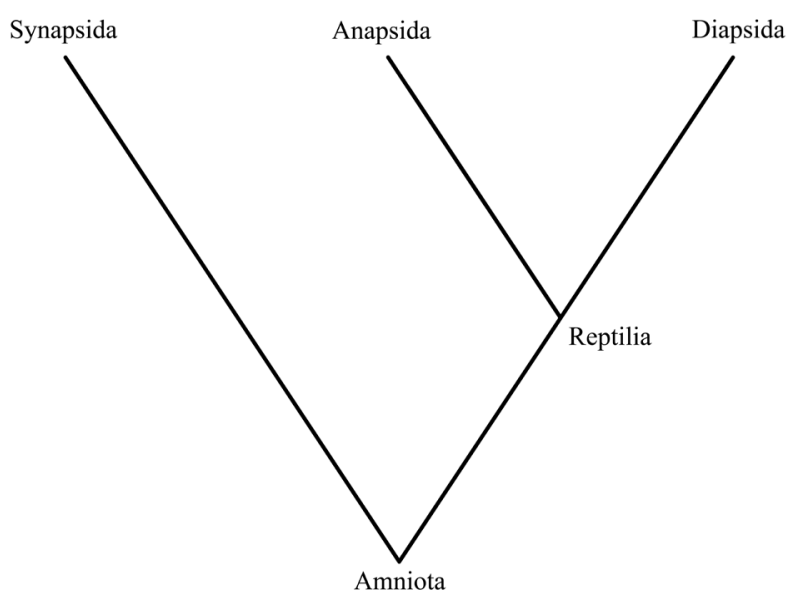

Figure 1. Phylogeny of Amniota, summarized from the proposal by Gauthier et al. (1988, Fig. 3). This proposal conserves the name Reptilia and redefines it as a monophyletic group that includes Aves, which pertains to the Diapsida clade. Both terminal branches include fossil and extant organisms (see Gauthier et al., 1988 for details).

Figura 1. Filogenia de los Amniota, resumida de la propuesta de Gauthier et al. (1988, Fig. 3). Esta propuesta conserva el nombre Reptilia y lo redefine como un grupo monofilético que incluye a las Aves que son parte del clado Diapsida. Ambas ramas terminales incluyen organismos fósiles y recientes (ver Gauthier et al. 1988 para más detalles).

La filogenia de Gauthier et al. (1988) fue sustituida por la que propusieron Laurin y Reisz (1995). Esta última filogenia agrupaba a los Amniota en dos grandes clados: Synapsida (que incluye a los mamíferos) y Sauropsida, que a su vez incluye a los Mesosauridae y Reptilia; en este último clado quedaron incluidos todos los reptiles vivientes y fósiles y las aves (Fig. 2). Laurin y Reisz (1995) también introdujeron dos nombres que se han vuelto de uso común en filogenias de los Amniota: Parareptilia y Eureptilia, los cuales fueron propuestos originalmente por Olson (1947). De acuerdo con Olson (1947), Parareptilia incluye a varios grupos fósiles que designó Diadecta y a Chelonia; en los Eureptilia al resto de los reptiles fósiles y vivientes (Captorhina, Parapsida, Euriapsida y Diapsida) y a los Synapsida. Sin entrar en una discusión de cómo ha variado el uso de estos dos términos, la cual está más allá del propósito de este comentario, las categorías Parareptilia y Eureptilia se han usado en casi todas las filogenia de los Amniota posteriores a Laurin y Reisz (1995), variando su contenido de grupos de reptiles fósiles conforme se ha avanzado en el conocimiento de los amniota.

No obstante lo anterior, Benton (1990a, 1991) usó el término Sauropsida en sustitución de Reptilia. Al hacer una revisión de la literatura lo insinúa en 1990a y lo formaliza en 1991 (Amniota
(Synapsida + nodo no nombrado [NN]), NN (Parareptilia + Sauropsida), conteniendo los Sauropsida a los Anapsida y Diapsida. Aunque Benton (1991) concluye que Synapsida y Sauropsida son taxones hermanos, lo cual es un error de interpretación de su dendrograma (Modesto \& Anderson, 2004). Posteriormente, introdujo el término Sauropsida en sustitución de Reptilia, en su libro de texto de Paleontología de Vertebrados, desde la primera edición (Benton, 1990b), utilizando el mismo esquema de su artículo de 1991. A partir de la segunda edición (1997) y hasta la tercera (Benton, 2005a), cambió su esquema de la primera edición, corrigiendo la inconsistencia de su propuesta original, en la segunda edición (Benton, 1997); Amniota (Synapsida +Sauropsida), Sauropsida (Mesosauridae + Eureptilia), Eureptilia (Anapsida + NN), NN incluye al resto de los reptiles conteniendo a las Aves. En la tercera edición de su libro (Benton, 2005a) adopta el siguiente esquema: Amniota (Synapsida + Sauropsida), Sauropsida (Anapsida $+\mathrm{NN}$ ). Los Anpasida incluyen a las tortugas varios grupos fósiles y los Mesosauridae; el NN incluye los otros reptiles fósiles, los diapsida que incluyen a las Aves. No obstante lo anterior, en la cuarta edición de este texto Benton (2015), abandonó su propuesta retomando que los Amniota incluyen a dos grupos Synapsida y Reptilia, el cual a su vez incluye a los Parareptilia

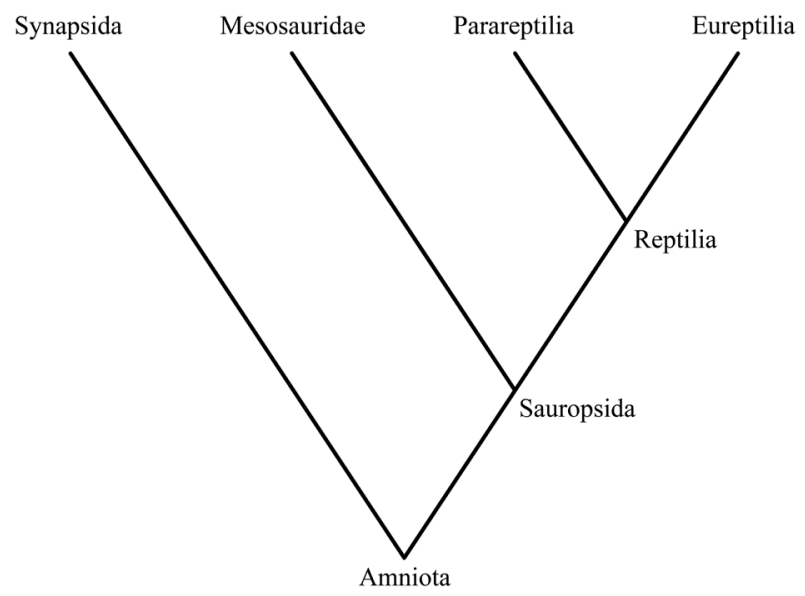

Figure 2. Phylogeny of Amniota, summarized from the proposal of Laurin and Reisz (1995, Fig. 2). The authors did not use the name Sauropsida as a substitute for Reptilia but rather include the Mesosauridea, whose position has been controversial (see details in Laurin and Reisz, 1995).

Figura 2. Filogenia de los Amniota, resumida de la propuesta de Laurin \& Reisz (1995, Fig. 2). Ellos no usaron el nombre Sauropsida como sustituto de Reptilia sino para incluir a los Mesosauridea, cuya posición ha sido controversial (ver detalles en Laurin \& Reisz, 1995). 
y los Eureptilia. Se puede consultar Benton (1990a) para una revisión de la literatura que utilizó para su propuesta.

Existe otro libro de texto, ampliamente usado, el cual lleva 10 ediciones, intitulado "Vertebrate Life". Las dos primeras por McFarland et al. $(1979,1985)$ y de la tercera a la última por Pough et al. (1989, 1996, 1999, 2002, 2005, 2008, 2013, 2018). Desde la primera edición de este libro de texto, se ha usado y explicado el uso del término Sauropsida, aunque no se usó para remplazar a Reptilia. Se explican las razones, similitudes morfológicas, por las que Huxley puso a reptiles y aves, bajo la misma categoría Sauropsida. En el índice de las dos primeras ediciones aparece la palabra "Reptiles" (McFarland et al., 1979, 1985). A partir de la tercera edición (Pough et al., 1989) desaparece la palabra "Reptiles" del índice, pero no se adopta, como sustituto de Reptilia a Sauropsida, solo se mencionan los grupos de reptiles sin ser referidos a una categoría superior y se explica el trabajo de Huxley en un contexto diferente, ubicando a las Aves como dinosaurios terópodos más derivados. No tuve acceso a la cuarta edición del libro de Pough y sus colaboradores (1996); pero en la quinta edición (Pough et al., 1999) se explica el trabajo de Huxley en el mismo contexto que en la tercera edición, agregando una crítica a la taxonomía tradicional de reconocer a las clases Reptilia y Aves al mismo nivel. Se incorpora a la discusión de las diferencias entre los Synapsida y Sauropsida aspectos fisiológicos y reconocen cuatro grupos principales de amniotas (p. 293): (Synapsida + Sauropsida + Parareptilia + Eureptilia). En la sexta edición Pough et al. (2002) adoptan el esquema de Laurin y Reisz (1995), ver figura 2. De la 8a edición a la 10ma (Pough et al., 2008, 2013, 2019) [no tuve acceso a la 7a edición, 2005] se introduce en el índice del libro a Sauropsida (Tortugas + Lepidosdaurios + Archosaurios) y se introduce el tema intitulado "Synapsids and Sauropsids: Two approaches to terrestrial life". En este capítulo del texto se hace un resumen de las diferencias anatómicas y fisiológicas de los grupos mencionados cuyo esquema de clasificación queda: Amniota (Synapsida + Sauropsida).

Harvey Pough aclara que seguirá usando el esquema de sustituir a Reptilia por Sauropsida, en la próxima edición se su texto de "Vertebrate Life", aunque admite que en su concepto de Sauropsida incluye a los Parareptilia (todos representados por grupos fósiles), para los cuales asume que debieron haber tenido el mismo sistema cardiovascular, renal y respiratorio que el resto de los reptiles y aves (Pough, com. pers., 5 abril del 2021); caracteres que usa para sostener a los Sauropsida, pero que no son observables en el registro fósil.

Adicionalmente, cabe mencionar que de los dos textos de herpetología de amplio uso que existen, ninguno utiliza el término de Sauropsida como sustituto de Reptilia. Llama la atención que las cuatro ediciones de "Herpetology" editado por Pough et al. (1998, 2001, 2004, 2016), el término ni siquiera se menciona y se utiliza a Reptilia. Esto contrasta con el uso que se le da a Sauropsida en el texto de Vertebrate Life, en el que Pough es el editor principal. Por otro lado, el texto de Vitt y Caldwell, en sus últimas dos ediciones $(2009,2014)$, usan el término de forma ambigua, pero no como un remplazo de Reptilia.

Otros trabajos, como el de Modesto (1999), estudiando la estructura de un mesosaurio (Stereosternum tumidum), concluye que el nombre Reptilia tiene prioridad sobre Sauropsida, en el sentido del principio de prioridad (aunque este principio no se aplica por arriba de las categoría de superfamilia; Ohl, 2007), y como un nombre en términos de nomenclatura filogenética (por haberse publicado primero). Cabe mencionar que Reptilia fue originalmente propuesto por Laurenti (1768) y como ya se mencionó Sauropsida por Huxley (1869). Para Modesto (1999), el término Reptilia fue usado en el contexto de la nomenclatura filogenética por primera ocasión por Gauthier et al. (1988). Modesto y Anderson (2004) hicieron una discusión amplia del tema incluyendo el problema que representa el uso de definiciones filogenética de taxones. Tratando de reconciliar el sistema tradicional, basado en el Código Internacional de Nomenclatura Zoológica (ICZN, 2000) y el PhyloCode (Cantino \& de Queiroz, 2020), proponen una definición de Reptilia y

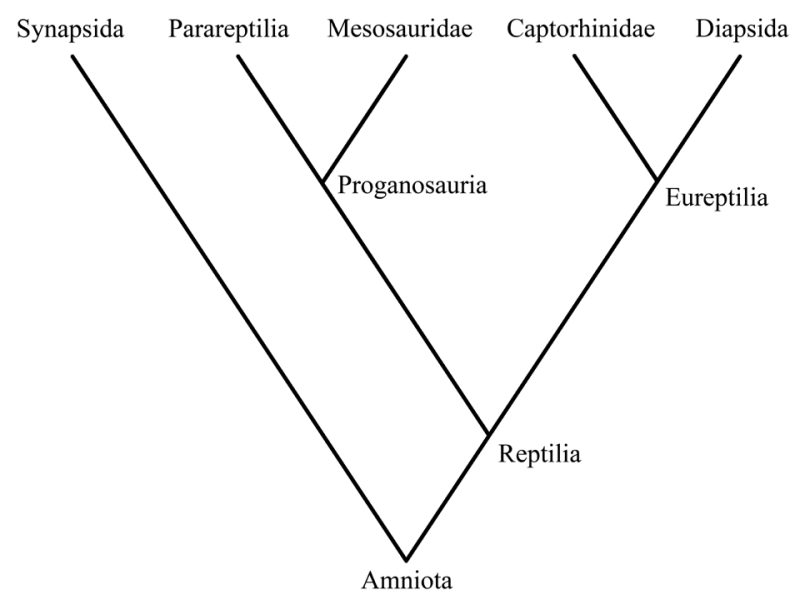

Figure 3. Phylogeny of Amniota, summarized from the proposal by Modesto and Anderson (2004, Fig. 3). This proposal includes turtles as diapsids and preserves the name Reptilia, applying criteria of the phylogenetic nomenclature.

Figura 3. Filogenia de los Amniota, resumida de la propuesta de Modesto y Anderson (2004, Fig. 3). Esta propuesta incluye a las tortugas como diápsidos y preserva el nombre de Reptilia, aplicando criterios de la nomenclatura filogenética. 
sugieren usar este término en lugar de Sauropisida (Fig. 3). Para una revisión de los diferentes usos de Sauropsida y Reptilia con base en los enfoques tradicional y filogenético ver el trabajo de Modesto y Anderson (2004).

Aunque el principio de prioridad no se aplica a taxones por arriba del nivel de familia, el término Reptilia es de amplio uso y conocimiento y fue propuesto casi 100 años antes que el término Sauropsida. Por ejemplo, Benton (1990c, 2005b), a pesar de haber intentado introducir el término Sauropsida como sustituto de Reptilia (Benton, 1991), en su libro de divulgación intitulado "The reign of the Reptiles" mantuvo el término más común y conocido, en lugar de intitular su libro "El reino de los Saurópsidos". Pough menciona que en la décimoprimera edición de su libro "Vetebrate Life", que aparecerá el próximo año, para los grupos recientes usará el término más familiar de Reptilia, y que en el caso de incluir a los grupos fósiles utilizará Sauropsida (H. Pough y W. Bemis, com. pers., 7 abril 2021).

Si estuviéramos de acuerdo con los preceptos del PhyloCode (Cantino \& de Queiroz, 2020), el uso del término Reptilia (un nombre basado en un nodo) cuya definición sería "el ancestro común de Chelonia, Squamata, Crocodylia y Aves y sus descendientes (J. McGuire, com. pers., 26 marzo, 2021), sigue siendo válido para referirse a los reptiles recientes (Fig. 4). El término Sauropsida (un nombre basado en una rama) se definiría como todos los taxones más cercanamente relacionados con los reptiles recientes que con los Synapsida (Fig. 4). Esto es que Sauropsida incluye a todos los Reptilia, incluyendo los taxones fósiles en la rama que lleva a Reptilia arriba del nodo de los Amniota (J. McGuire, com. pers., 26 marzo, 2021). Con esta propuesta y de acuerdo con los proponentes del PhyloCode, esta nomenclatura debería traer más estabilidad a los nombres que la nomenclatura linneana; no obstante, lo que hace es introducir dos nombres para el mismo grupo de organismos.

No veo la simplificación y como ya lo demostraron Gauthier et al. (1988) y Modesto y Anderson (2004), se pueden adoptar los principios de la nomenclatura filogenética y preservar el nombre más antiguo y conocido, incluso cambiando las definiciones de dichos nodos y ramas, las cuales han cambiado con el descubrimiento de nuevos fósiles o nuevos caracteres y su reinterpretación (Dominguez \& Wheeler, 1997; Modesto \& Anderson, 2004). Por ejemplo, ver cómo ha cambiado la posición de los Chelonia de ser considerados anápsidos a diápsidos, utilizando una reinterpretación de la fenestración del cráneo y caracteres moleculares (ver revisión en Zardoya \& Meyer, 2001 y Lee, 2013) y ver cómo ha afectado esto en la filogenia de los Amniota (Modesto \& Anderson, 2004).

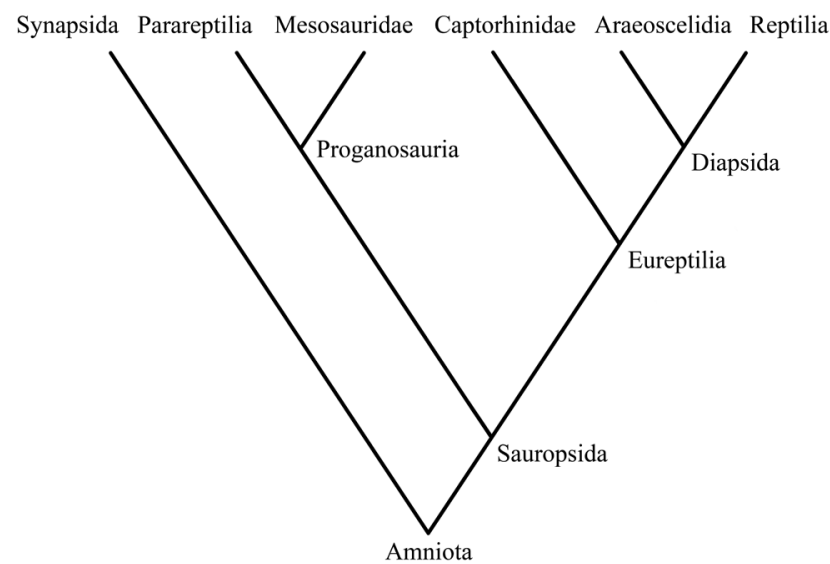

Figure 4. Phylogeny of Amniota, summarized from the proposal of Modesto and Anderson (2004, Fig. 2). This proposal includes turtles as diapsids using phylogenetic definitions, leaving Reptilia as a crown group, including only recent reptiles (see details in Modesto \& Anderson, 2004).

Figura 4. Filogenia de los Amniota, resumida de la propuesta de Modesto y Anderson (2004, Fig. 2). Esta propuesta incluye a las tortugas como diápsidos usando definiciones filogenéticas, por lo que los Reptilia quedan como un grupo corona, incluyendo solo a reptiles recientes (ver detalles en Modesto \& Anderson, 2004).

Para entender los conceptos de la nomenclatura filogenética ver Morrone (2013). Para las críticas a la propuesta del PhyloCode, ver Dominguez y Wheeler (1997), Nixon y Carpenter (2000), Keller et al. (2003), Nixon et al. (2003), Wheeler (2004) y Platnick (2012).

Puedo concluir que el uso del término Sauropsida para remplazar a Reptilia en las clasificaciones solo puede conducir a confusión y a abandonar un concepto de amplio uso entre herpetólogos, otros biólogos y el público en general. En la revisión que se ha presentado (sin pretender que sea totalmente exhaustiva) se ha demostrado que el término Sauropsida se ha usado de forma obscura e inconsistente (Benton, 1990b, 1997, 2005a; F. H. Pough com. pers., 5 de abril del 2021) en la literatura de la taxonomía de los reptiles y que existe un consenso entre la mayoría de los especialistas en no usarlo como sustituto de Reptilia. Su uso en otros trabajos se ha justificado con base en una revisión superficial de la literatura y una interpretación errónea de la misma (Manríquez-Morán et al., 2017). Se ha referido a este térmico bajo categorías linneanas incorrectas y que reflejan una taxonomía inconsistente (Torres-Torres et al., 2017). También ha sido usado en trabajos de no especialistas, posiblemente basados en uno de los dos únicos textos que lo ha 
usado como remplazo de Reptilia, o sin justificar la adopción del término en lugar de Reptilia (Fernández-Badillo et al., 2016; Burgos Gallardo et al., 2020; Griebeler \& Wener, 2018; EcuRed; Pérez, 2021; Pollen et al., 2009).

En resumen, no encuentro justificación válida, desde el punto de vista de la nomenclatura, ya sea tradicional o filogenética, ni desde el punto de vista práctico para continuar con el uso del término Sauropsida en sustitución de Reptilia. Esta práctica debe ser abandonada para evitar la proliferación de nombres incorrectamente aplicados.

Agradecimientos.- A Jimmy McGuire, Lee Fitzgerald y Jack Sites, quienes enseñan herpetología en sus respectivas universidades, por sus puntos de vista. A Harvey Pough por aclarar el uso del término Sauropsida en su libro de texto "Vertebrate Life", aunque sigo en desacuerdo con él. A Martha L. Crump por facilitar la comunicación con Harvey Pough. A Laurie Vitt por sus comentarios con relación al uso del término Sauropsida. A Adriana J. X. González-Hernández, Juana M. Garza-Castro y Carlos J. Balderas-Valdivia, por estimular este comentario. A Alicia Villela por su ayuda y a Juan J. Morrone por su revisión del manuscrito. Al Sr. Víctor Hernández López de la Biblioteca "Ricardo Monjes López" de la Facultad de Ciencias, UNAM.

\section{LITERATURA CITADA}

Baur, G. 1887. On the phylogenetic arrangement of the Sauropsida. Journal of Morphology 1:93-104.

Benton, M.J. 1990a. Phylogeny of the major Tetrapod groups: Morphological data and divergence dates. Journal of Molecular Evolution 30:409-424.

Benton, M.J. 1990b. Vertebrate paleontology, biology and evolution. Harper Collins Academic, London, UK.

Benton, M.J. 1990c. The reign of the reptiles. Crescent Books, New York, USA.

Benton, M.J. 1991. Amniote phylogeny. Pp. 317-330. En: H. P. Schultze y L. Trueb (Eds.). Origins of the higher groups of Tetrapods, controversy and consensus. Comstock Publishing Associates, Ithaca, New York, USA.

Benton, M.J. 1997. Vertebrate paleontology 2th Edit. Blackwell Science, Massachusetts, USA [tercera reimpresión 2003].
Benton, M.J. 2005a. Vertebrate paleontology, 3th Edit. Blackwell Publishing Ltd. Oxford, UK.

Benton, M.J. 2005b. The reign of the reptiles. Reprinted by Quantum Publishing Ltd. London, UK.

Benton, M.J. 2015. Vertebrate paleontology, 4th Edit. Wiley and Sons, Sussex UK.

Burgos Gallardo, F., J.L. Baldo y J.D. Baldo. 2020. Taxocenosis de las Serpientes (Sauropsida: Squamata) de la provincia de Jujuy, Argentina. Cuadernos de Herpetología 34:53-77.

Cantino , P. D. \& K. de Queiroz. 2020. PhyloCode, Version 6. http:// phylonames.org/code/ [Consultado en marzo del 2021].

Dominguez, E. \& Q. D. Wheeler. 1997. Taxonomic stability is ignorance. Cladistics, 13:367-372.

EcuRed: Sauropsida [https://www.ecured.cu/ [Consultado en marzo 2021].

Fernández-Badillo, L., N.L. Manríquez-Morán, J.M. Castillo-Cerón. \& I. Goyenechea. 2016. Análisis herpetofaunístico de la zona árida del estado de Hidalgo. Revista Mexicana de Biodiversidad 87:156-170.

Flores-Villela, O. \& A. Navarro S. 1993. Clasificación actual de los amniota. Número Especial 7, Biología y problemática de los vertebrados en México. CIENCIAS, revista de difusión de la Facultad de Ciencias de la UNAM:63-69.

Gauthier, J., A.G. Kluge \& T. Rowe, 1988. Amniote phylogeny and the importance of fossils. Cladistics 4:105-209.

Goodrich, E.S. 1916. On the classification of Reptilia. Proceeding of the Royal Society of London B 89:261-276.

Griebeler, E.M. \& J. Wener. 2018. Formal comment on: Myhrvold (2016) Dinosaur metabolism and the allometry of maximum growth rate. PLoS ONE 11: e0163205.

Hennig, W. 1968. Elementos de una sistemática filogenética. Editorial Universitaria de Buenos Aires, Argentina.

Hennig, W. 1975. Cladistic analysis or cladistic classification: A reply to Ernst Mayr. Systematic Zoology 24:244-256. 
Huxley, T.H. 1864. Lectures on the elements of comparative anatomy, on the classification of animals and on the vertebrate skull. John Churchill and Sons. London, UK.

Huxley, T.H. 1869. An introduction to the classification of animals. John Churchill and Sons. London, UK.

Huxley, T.H. 1870. On the classification of the Dinosauria, with observations on the Dinosauria of the Trias. Quarterly Journal of the Geological Society, London 26:32-50.

Huxley, T.H. 1872. A manual of the anatomy of vertebrated animals. D. Appleton and Company, Brodway, UK.

International Commission of Zoological Nomenclature. 2000. International Code of Zoological Nomenclature, 4th edit. https:// www.iczn.org/the-code/the-international-code-of-zoologicalnomenclature/the-code-online/ [Consultado marzo del 2021].

Keller, R.A., R.N. Boyd \& Q.D. Wheeler. 2003. The illogical basis of phylogenetic nomenclature. The Botanical Review 69:93-110.

Kluge, A. G. 1990. On the special treatment of fossils and taxonomic burden: A response to Loconte. Cladistics 6:187-190.

Laurenti, J.N. 1768. Specimen medicum, exhibens synopsis Reptilium emendatum, cum experimentis circa venena et antidote Reptilium Austriacorum. Joan Thom., Nob, de Trattnern, Vienna.

Laurin, M. \& R.R. Reisz. 1995. A reevaluation of early amniote phylogeny. Zoological Journal of the Linnaean Society 113:165-223.

Lee, M.S.Y. 2013. Paleontology: Turtles in transition. Current Biology 23:R513-R515

Loconte, H. 1990. Cladistic classification of Amniota: a response to Gauthier et al. Cladistics 6:187-190.

Manríquez-Morán, N.L., J.M. Castillo-Cerón, I. Goyenechea MayerGoyenechea, R. Cruz-Elizalde, U. Hernández-Salinas, D. LaraTufiño, L.M. Badillo-Saldaña, C. Berriozabal-Islas \& A. RamírezBautista. 2017. Riqueza y biodiversidad de saurópsidos (no Aves) del Estado de Hidalgo. Pp. 505-528. En: A. Ramírez-Bautista, A. Sánchez-González, G. Sánchez-Rojas \& C. Cuevas-Cardona (eds.). Biodiversidad del estado de Hidalgo. Tomo I. Universidad Autónoma del Estado de Hidalgo/Consejo Nacional de Ciencia y Tecnología. Pachuca de Soto, Hidalgo, México.

REVISTA LATINOAMERICANA DE HERPETOLOGÍA Vol.04 No.01 / Mayo 2021
McFarland, W.N., F.H. Pough, T.J. Cade \& J.B. Heiser. 1979. Vertebrate life. Collier Macmillan International Editions, New York, New York, USA.

McFarland, W.N., F.H. Pough, T.J. Cade \& J.B. Heiser. 1985. Vertebrate Life, 2th edit. Macmillan Publishing Co. New York, New York, USA.

Modesto, S.P. 1999. Observations on the structure of the early Permian reptile Stereosternum tumidum Cope. Paleontologia Africana 35:7-19.

Modesto, S.P. \& J.S. Anderson. 2004. The phylogenetic definition of Reptilia. Systematic Biology 53:815-821.

Morrone, J.J. 2013. Sistemática: Fundamentos, métodos, aplicaciones. Facultad de Ciencias, UNAM, Ciudad de México, México.

Nixon, K.C. \& J.M. Carpenter. 2000. On the other "phylogenetic systematics". Cladistics 16:298-318.

Nixon, K.C., J.M. Carpenter, \& D.W. Stevenson. 2003. The PhyloCode is fatally flawed, and the "Linnean" system easily be fixed. Botanical Review 69:111-120.

Ohl, M. 2007. Principles of taxonomy and current classification: Current procedures for naming and classifying organisms. Pp. 141-166. En W. Henke \& I. Tattersall (Eds.). Handbook of Paleoanthropology, Volume I Principles, methods and approaches. Springer-Verlag, Berlin, Alemania.

Olson, E.C. 1947. The family Diadectidae and its bearing on the classification of Reptiles. Fieldiana Geology 11:3-53.

Pearse, A.S. (ed.) 1947. Zoological names: A list of phyla, classes, and orders. Prepared for Section F, American Association for the Advancement of Science. 2th edit. Durham, North Carolina, U.S.A.

Pérez, J.I. 2021. Cuaderno de Cultura Cientifica https:// culturacientifica.com/2017/10/24/sistemas-respiratorios-lospulmones-reptiles-aves/ [Consultado marzo 2021].

Platnick, N.I. 2012. The poverty of the PhyloCode: A reply to de Queiroz and Donoghue. Systematic Biology, 61:360-361.

Pollen, A.A., A.F.P. Cheung \& Z. Molnár. 2009. Evolution and embryological development of the cortex in Amniotes. En: M.D. 
Binder, N. Hirokawa \& U. Windhorst (eds.), Encyclopedia of neuroscience. Springer, Berlin, Heidelberg.

Pough, F.H., J.B. Heiser \& W.N. McFarland. 1989. Vertebrate Life 3th Edit. Macmillan Publishing Co. New York, New York, USA.

Pough, F.H., C.M. Janis \& J.B. Heiser. 1999. Vertebrate Life 5th Edit. Prentice Hall, New Jersey, USA.

Pough, F.H., C.M. Janis \& J.B. Heiser. 2002. Vertebrate Life 6th Edit. Prentice Hall, New Jersey, USA.

Pough, F.H., C.M. Janis \& J.B. Heiser. 2008 Vertebrate Life 8th Edit. Benjamin Cummings Publishing Company, San Francisco, California, USA.

Pough, F.H., C.M. Janis \& J.B. Heiser. 2013. Vertebrate Life 9th Edit. Pearson, Boston, Massachusetts, USA.

Pough, F.H. \& C.M. Janis. 2018. Vertebrate Life 10th Edit. Sinauer Associates, New York, New York, USA.

Pough F.H., R.M. Andrews, J.E. Cadle, M.L. Crump, A.H. Savitzky \& K.D. Wells. 1998. Herpetology. Prentice Hall, New Jersey, USA.

Pough F.H., R.M. Andrews, J.E. Cadle, M.L. Crump, A.H. Savitzky \& K.D. Wells. 2001. Herpetology, 2th Edit. Prentice Hall, New Jersey, USA.

Pough F.H., R.M. Andrews, J.E. Cadle, M.L. Crump, A.H. Savitzky \& K.D. Wells. 2004. Herpetology, 3th Edit. Pewarsons, Prentice Hall, New Jersey, USA.
Pough F.H., R.M. Andrews, M.L. Crump, A.H. Savitzky, K.D. Wells \& M.C. Brandley. 2016. Herpetology, 4th Edit. Sinauer, Sunderland, Massachusetts, USA.

Switek, B. 2010. Thomas Henry Huxley and the reptile to bird transition. En R. T. J. Moody, E. Buffetaut, D. Naish, \& D.M. Martill (eds), Dinosaurs and other extinct saurians: A historical perspective. Geological Society, London, Special Publications 343:251-263.

Torres Torres, A.M., C. Trespalacios, N.A. Pachón Barbosa \& N. Ruda. 2017. Diafanización como alternativa metodológica para el estudio anatómico en reptiles de la clase Sauropsida. Número Extraordinario, IV Congreso Nacional de Investigación en Enseñanza de la Biología.

Vitt, L.J. \& J.P. Caldwell. 2009. Herpetology, an introductory biology of amphibians and reptiles 3th Edit. Elsevier-Academic Press, Amsterdam, Países Bajos.

Vitt, L.J. \& J.P. Caldwell. 2014. Herpetology, an introductory biology of amphibians and reptiles 4th Edit. Elsevier-Academic Press, Amsterdam, Países Bajos.

Watson, D.M.S. 1957. On Millerosaurus and the early history of the Sauropsid reptiles. Philosophical Transactions of the Royal Society of London. Series B 240:325-400.

Wheeler, Q.D. 2004. Taxonomic triage and the poverty of phylogeny. Philosophical Transactions of the Royal Society of London B 359:571-583.

Zardoya, R. \& A. Meyer. 2001. The evolutionary position of turtles revised. Naturwissenschaften 88:193-200. 